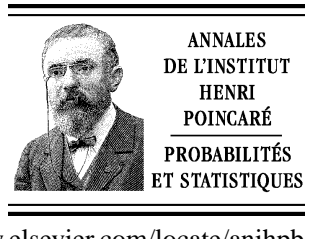

www.elsevier.com/locate/anihpb

\title{
Law of large numbers for a class of superdiffusions
}

\author{
János Engländer ${ }^{\mathrm{a}, *}$, Anita Winter ${ }^{\mathrm{b}}$ \\ a Department of Statistics and Applied Probability, University of California, Santa Barbara, CA 93106-3110, USA \\ $\mathrm{b}$ Mathematisches Institut, Universität Erlangen-Nürnberg, Bismarckstraße 1 1/2, 91054 Erlangen, Germany
}

Received 1 March 2004; received in revised form 11 January 2005; accepted 15 March 2005

Available online 26 September 2005

\begin{abstract}
Pinsky [R.G. Pinsky, Transience, recurrence and local extinction properties of the support for supercritical finite measurevalued diffusions, Ann. Probab. 24 (1) 237-267] proved that the finite mass superdiffusion $X$ corresponding to the semilinear operator $L u+\beta u-\alpha u^{2}$ exhibits local extinction if and only if $\lambda_{c} \leqslant 0$, where $\lambda_{c}:=\lambda_{c}(L+\beta)$ is the generalized principal eigenvalue of $L+\beta$ on $\mathbb{R}^{d}$. For the case when $\lambda_{c}>0$, it has been shown in Engländer and Turaev [J. Engländer, D. Turaev, A scaling limit theorem for a class of superdiffusions, Ann. Probab. 30 (2) 683-722] that in law the superdiffusion locally behaves like $\exp \left[t \lambda_{c}\right]$ times a non-negative non-degenerate random variable, provided that the operator $L+\beta-\lambda_{c}$ satisfies a certain spectral condition ('product-criticality'), and that $\alpha$ and $\mu=X_{0}$ are 'not too large'.

In this article we will prove that the convergence in law used in the formulation in [J. Engländer, D. Turaev, A scaling limit theorem for a class of superdiffusions, Ann. Probab. 30 (2) 683-722] can actually be replaced by convergence in probability. Furthermore, instead of $\mathbb{R}^{d}$ we will consider a general Euclidean domain $D \subseteq \mathbb{R}^{d}$.

As far as the proof of our main theorem is concerned, the heavy analytic method of [J. Engländer, D. Turaev, A scaling limit theorem for a class of superdiffusions, Ann. Probab. 30 (2) 683-722] is replaced by a different, simpler and more probabilistic one. We introduce a space-time weighted superprocess ( $H$-transformed superprocess) and use it in the proof along with some elementary probabilistic arguments.
\end{abstract}

○ 2005 Elsevier SAS. All rights reserved.

\section{Résumé}

Pinsky [R.G. Pinsky, Transience, recurrence and local extinction properties of the support for supercritical finite measurevalued diffusions, Ann. Probab. 24 (1) 237-267] a prouvé que le processus de superdiffusion de masse finie $X$ correspondant á l'operateur semilinéaire $L u+\beta u-\alpha u^{2}$ possède la propriété d'extinction locale si, et seulement si, $\lambda_{c} \leqslant 0$, où $\lambda_{c}:=\lambda_{c}(L+\beta)$ est la valeur propre principale généralisée de $L+\beta$ dans $\mathbb{R}^{d}$. Dans le cas où $\lambda_{c}>0$, et pour un opérateur $L+\beta-\lambda_{c}$ satisfaisant une condition spectrale (de 'criticalité-produit'), et pourvu que $\alpha$ et $\mu=X_{0}$ ne soient pas trop grands, Engländer and Turaev [J. Engländer, D. Turaev, A scaling limit theorem for a class of superdiffusions, Ann. Probab. 30 (2) 683-722] ont montré le processus se comporte localement et en loi comme $\exp \left[t \lambda_{c}\right]$ avec une constante multiplicative aléatoire non dégénérée.

\footnotetext{
* Corresponding author.

E-mail addresses: englander@pstat.ucsb.edu (J. Engländer), winter@mi.uni-erlangen.de (A. Winter).
} 
Dans cet article, nous montrons que la convergence en loi de [J. Engländer, D. Turaev, A scaling limit theorem for a class of superdiffusions, Ann. Probab. 30 (2) 683-722] peut être renforcée en la convergence en probabilité. De plus, l'espace $\mathbb{R}^{d}$ peut étre remplacé par un domaine euclidien quelconque.

S'agissant de la preuve du theorème principal la lourde methode analytique de [J. Engländer, D. Turaev, A scaling limit theorem for a class of superdiffusions, Ann. Probab. 30 (2) 683-722] est remplacée par une approche probabiliste plus simple. Nous introduisons une renormalisation spatio-temporelle du superprocessus ('H-transformed superprocess') que nous utilisons dans la preuve combinée a des arguments probabilistes élémentaires.

(c) 2005 Elsevier SAS. All rights reserved.

MSC: $60 \mathrm{~J} 60 ; 60 \mathrm{~J} 80$

Keywords: Super-Brownian motion; Superdiffusion; Superprocess; Law of Large Numbers; $H$-transform; Weighted superprocess; Scaling limit; Local extinction; Local survival

\section{Introduction}

\subsection{Preparation}

We consider a superprocess arising as the short life time and high density diffusion limit of a branching particle system, which can be described as follows: in the $n$th approximation step each particle has mass $1 / n$ and lives a random time which is exponential with mean $1 / n$. While a particle is alive, its motion is described by a diffusion process corresponding to the operator $L$. At the end of its life, the particle dies and is replaced by a random number of particles situated at the parent particle's final position. The distribution law of the number of descendants is spatially varying such that the mean number of descendants is $1+\beta(x) / n$, while the variance is assumed to be $2 \alpha(x)$. All these mechanisms are independent of each other. The process is determined by the quadruple $(L, \beta, \alpha ; D)$, where $L$ is a second order elliptic operator corresponding to the underlying diffusion process on $D$. See Appendix A in Engländer and Pinsky [7] for a precise statement on the particle approximation.

We start by presenting a formal description of the model considered in this article. For convenience we first recall the basic notation: let $D \subseteq \mathbb{R}^{d}$ be a domain and let $\mathcal{B}(D)$ denote the Borel sets of $D$. We write $\mathcal{M}_{f}(D)$ and $\mathcal{M}_{c}(D)$ for the class of finite measures resp. the class of finite measures with compact support on $\mathcal{B}(D)$. For $\mu \in \mathcal{M}_{f}(D)$, denote $\|\mu\|:=\mu(D)$ and let $C_{b}^{+}(D)$ and $C_{c}^{+}(D)$ be the class of non-negative bounded continuous resp. non-negative continuous functions $D \rightarrow \mathbb{R}$ having compact support. Write $C^{k, \eta}(D)$ for the usual Hölder spaces of index $\eta \in(0,1]$ including derivatives of order $k$, and set $C^{\eta}(D):=C^{0, \eta}(D)$.

We continue with the definition of the $(L, \beta, \alpha ; D)$-superdiffusion, $X$. Let $L$ be an elliptic operator on the domain $D \subseteq \mathbb{R}^{d}$ of the form

$$
L:=\frac{1}{2} \nabla \cdot a \nabla+b \cdot \nabla,
$$

where $a_{i, j}, b_{i} \in C^{1, \eta}(D), i, j=1, \ldots, d$, for some $\eta \in(0,1]$, and the matrix $a(x):=\left(a_{i, j}(x)\right)$ is symmetric, and positive definite for all $x \in D$. In addition, let $\alpha, \beta \in C^{\eta}(D)$, and assume that $\alpha$ is positive, and $\beta$ is bounded from above.

We now present our model.

Definition 1 (Time-homogeneous superdiffusion). Let $\left(X, \mathbf{P}^{\mu}, \mu \in \mathcal{M}_{f}(D)\right)$ denote the $(L, \beta, \alpha ; D)$-superdiffusion. That is, $X$ is the unique $\mathcal{M}_{f}(D)$-valued continuous (time-homogeneous) Markov process which satisfies, for any $g \in C_{b}^{+}(D)$,

$$
\mathbf{E}^{\mu} \exp \left\langle X_{t},-g\right\rangle=\exp \langle\mu,-u(\cdot, t)\rangle
$$


where $u$ is the minimal non-negative solution to

$$
\left.\begin{array}{l}
u_{t}=L u+\beta u-\alpha u^{2} \quad \text { on } D \times(0, \infty), \\
\lim _{t \downarrow 0} u(\cdot, t)=g(\cdot) .
\end{array}\right\}
$$

As usual, $\langle v, f\rangle$ denotes the integral $\int_{D} v(\mathrm{~d} x) f(x)$.

(See Dynkin [4,5] or Dawson [2] for the definition of superprocesses in general; see Engländer and Pinsky [7] for more on the definition in the particular setting above.)

Remark 1 (Time-inhomogeneous superdiffusion). The model under consideration is a time-homogeneous process. However, it is important to point out that for the formulation of the main theorem and the proof, the introduction of certain time-inhomogeneous superdiffusions is required. The previous definition will therefore be generalized for time-inhomogeneous superdiffusions in Appendix B. (See Definition 2.)

Let

$$
\lambda_{c}:=\lambda_{c}(L+\beta):=\inf \{\lambda \in \mathbb{R}: \exists u>0 \text { satisfying }(L+\beta-\lambda) u=0 \text { in } D\}
$$

denote the generalized principal eigenvalue for $L+\beta$ on $D$. Let $\xi^{L}$ be the diffusion process on $D$ corresponding to $L$, and denote by $\mathbb{P}^{x}$ the law of $\xi^{L}$ starting at $x \in D$. Then from a probabilistic point of view, the generalized principal eigenvalue can be equivalently expressed as

$$
\lambda_{c}=\sup _{\left\{A: A \Subset D, \partial A \text { is } C^{2, \eta}\right\}} \lim _{t \rightarrow \infty} \frac{1}{t} \log \mathbb{E}^{x}\left[\exp \left[\int_{0}^{t} \beta\left(\xi_{s}^{L}\right) \mathrm{d} s\right] ; \tau^{A}>t\right],
$$

for any $x \in D$, where $\tau^{A}=\inf \left\{t \geqslant 0: \xi^{L}(t) \notin A\right\}$, and the $C^{2, \eta}$-boundary is defined with the help of $C^{2, \eta}$-maps in the usual way. (See Section 4.4 in Pinsky [15] on the subject). Hence, since $\beta$ is bounded from above, $\lambda_{c}<\infty$; and it is known from standard theory that for any $\lambda \geqslant \lambda_{c}$, there exists a function $0<f \in C^{2, \eta}(D)$ such that $(L+\beta) f=\lambda f$ on $D$. (See Section 4.3 in Pinsky [15].)

Pinsky proved that $X$ exhibits local extinction (i.e., the support of $X$ leaves any given bounded set, $\mathbf{P}^{\mu}$-a.s. for each $\mu \in \mathcal{M}_{c}$ ) if and only if $\lambda_{c} \leqslant 0$. (See Theorem 6 and Remark 1 in Pinsky [16].)

From now on we are interested in the situation where $X$ does not exhibit local extinction. We therefore assume that $\lambda_{c}>0$. We get a first rough impression about the local growth rate by the following statement taken from Theorem 7(b) in Pinsky [16]:

Lemma 0 (Local behavior in expectation). For $\mu \in \mathcal{M}_{c}(D)$, and $g \in C_{c}^{+}(D)$, satisfying $\|\mu\| \neq 0$ and $g \not \equiv$,

$$
\limsup _{t \rightarrow \infty} \exp [-\rho t] \mathbf{E}^{\mu}\left[\left\langle X_{t}, g\right\rangle\right]= \begin{cases}0 & \text { if } \rho>\lambda_{c}, \\ \infty & \text { if } \rho<\lambda_{c} .\end{cases}
$$

(See also Appendix A.)

We are therefore going to concentrate on scaling with the exponent $\rho=\lambda_{c}$. In addition to the concept of the generalized principal eigenvalue we will then need some further ones from the so-called criticality theory of second order elliptic operators. In particular, we will use the concepts of critical and product-critical (or product- $L^{1}$ critical) operators. Recall that the operator $L+\beta-\lambda_{c}$ is called critical if there exists a positive function $f$ satisfying that $\left(L+\beta-\lambda_{c}\right) f=0$ but there is no (minimal positive) Green's function for the operator $L+\beta-\lambda_{c}$. In this case $f$ is unique up to constant multiples and is called the ground state. The operator $L+\beta-\lambda_{c}$ is called product-critical if it is critical with ground state $0<\phi_{c}$, and $\phi_{c}$ and $\tilde{\phi}_{c}$ (i.e. the ground state for the formal adjoint of $L+\beta-\lambda_{c}$ ) satisfy $\left\langle\mathrm{d} x, \phi_{c} \tilde{\phi}_{c}\right\rangle<\infty$. In this case we normalize them by $\left\langle\mathrm{d} x, \phi_{c} \tilde{\phi}_{c}\right\rangle=1$. 
If $L+\beta-\lambda_{c}$ possesses a Green's function, then it is called subcritical.

For the reader, it will be handy to have Appendix 2 of [9] at hand, where a review on criticality theory is given. For a complete presentation of the theory, the reader is referred to Chapter 4 in [15].

\subsection{Motivation}

When $D=\mathbb{R}^{d}$ and $L+\beta-\lambda_{c}$ is product-critical, it is known from Theorem 1 in [9] that if $\left\|\alpha \phi_{c}\right\|_{\infty}<\infty$ and the initial state $\mu$ is such that $\left\langle\mu, \phi_{c}\right\rangle<\infty$, then the following holds in the vague topology:

$$
\lim _{t \rightarrow \infty} \exp \left[-\lambda_{c} t\right] X_{t}(\mathrm{~d} x)=N^{\mu} \tilde{\phi}_{c} \mathrm{~d} x \quad \text { in law, }
$$

where the limiting non-negative non-degenerate random variable $N^{\mu}$ was identified with the help of a certain invariant curve.

It is important to point out that even though product-criticality is equivalent to the ergodicity of an auxiliary diffusion process (see next section), the original motion process corresponding to L does not have to be ergodic. In fact it can even be transient - see Example 23 in [9].

(On the other hand, it follows from the discussion in Appendix A that when $L+\beta-\lambda_{c}$ on $D \subseteq \mathbb{R}^{d}$ is not product-critical, then for $\mu \in \mathcal{M}_{c}$, and $g \in C_{c}^{+}(D)$,

$$
\lim _{t \rightarrow \infty} \exp \left[-\lambda_{c} t\right]\left\langle X_{t}, g\right\rangle=0 \quad \text { in } L^{1} \text {.) }
$$

There are two disadvantages of the method used in [9]. First, the assumption that $D=\mathbb{R}^{d}$ is essential. Second, the proof does not yield any probabilistic insight.

In this paper our goal is to improve the statement in (7) and to provide a proof that is probabilistic in nature. We will show that the 'Law of Large Numbers' holds, that is, that one can replace the convergence in law by convergence in probability. ${ }^{1}$ Furthermore we will drop the assumption that $D=\mathbb{R}^{d}$. In the proof we will replace the analytic reasoning given in [9] (which relies on dynamical systems) by a more probabilistic one using spacetime weighted superprocesses ( $H$-transforms).

We suspect that in fact the Strong Law of Large Numbers holds, that is that the convergence in probability can be replaced by almost sure convergence. However we could not upgrade the proof of this paper to give the Strong Law.

In the recent paper [11] the authors study a supercritical superprocess taking values in the space of finite measures on $[0,1]$, whose underlying motion is the Wright-Fisher diffusion corresponding to the operator

$$
L=\frac{1}{2} x(1-x) \frac{\mathrm{d}^{2}}{\mathrm{~d} x^{2}}
$$

and whose branching mechanism is $\gamma u(1-u)$ with $\gamma>0$ (that is, $\alpha=\beta=\gamma$ ). They establish a dichotomy in the long-time behavior of this superprocess. For $\gamma \leqslant 1$, the mass in the interior $(0,1)$ dies out after a finite random time, while for $\gamma>1$, the mass in $(0,1)$ grows exponentially with rate $\gamma-1$ (as $t \rightarrow \infty$ and with positive probability) and is approximately uniformly distributed over $(0,1)$.

This result is in line with that of [9] if one considers the restriction of the superprocess on the (open) domain $(0,1)$. In fact it is easy to show that $\lambda_{c}:=\gamma-1$ is the principal eigenvalue of the linearized elliptic operator $L+\gamma$. Here is a possible argument: the operator $L+\gamma-\lambda_{c}=L+1$ can be $h$-transformed $(h=v$, where $v$ is an explicitly given function in the paper) into a diffusion operator, which - according to their Lemma 20 corresponds to a (positive) recurrent diffusion. Consequently, this $h$-transformed operator is critical, and thus its principal eigenvalue is zero. By $h$-transform invariance, the same is then true for the original operator $L+\gamma-\lambda_{c}$. (See again Chapter 4 in [15]). Furthermore, the product-criticality and boundedness assumptions are automatically satisfied by the boundedness of $D=(0,1)$.

\footnotetext{
1 Since the limit is not constant, therefore, unlike in classical probability theory, one has to distinguish between convergence in law (WLLN) and convergence in probability (LLN).
} 
Finally the fact that the limiting measure is the Lebesgue measure, is also in line with [9]. Indeed, according to [9], the limiting density is a harmonic function with respect to the adjoint of $L+\gamma-\lambda_{c}=L+1$, that is with respect to $\tilde{L}+1$, where $\tilde{L}$ is the adjoint of $L$. An easy computation reveals that

$$
\tilde{L}+1=\frac{1}{2} x(1-x) \frac{\mathrm{d}^{2}}{\mathrm{~d} x^{2}}+(1-2 x) \frac{\mathrm{d}}{\mathrm{d} x} .
$$

Since the adjoint of a critical operator is also critical, and since positive harmonic functions for a critical operator are unique up to constant multiples, the limiting density must be a properly normalized constant on the unit interval, that is, the limiting density is one.

However, as the authors point out referring to [9], 'their methods use in an essential way the fact that their underlying space is $\mathbb{R}^{d}$ (and not an open subset of $\mathbb{R}^{d}$, like $(0,1)$ ), and therefore their results are not applicable to our situation.'

In the present article, as already mentioned, we manage to overcome this difficulty, so the result of [11] will fit our main result. (In [11] the result is somewhat stronger as they prove convergence in $L^{2}$.)

\section{Main result}

Recall from (4) the definition of the principal eigenvalue $\lambda_{c}$ of $L+\beta$ on $D$ and the corresponding ground state $\phi_{c}$, and that throughout the paper we assume that $\lambda_{c}>0$. Also, $\left\{\mathcal{S}_{s}\right\}_{s} \geqslant 0$ will denote the semigroup ("expectation semigroup') corresponding to the operator $L+\beta$ on $D$. So far we have recalled (7). In order to replace in (7) the convergence in law by convergence in probability, we will assume the same conditions as in [9], except that we work with a generic domain $D$.

Assumption 1. In addition to the assumption that $\lambda_{c}>0$, also assume that $L+\beta-\lambda_{c}$ is product-critical, that $\alpha \phi_{c}$ is bounded and that $X$ starts in a state $\mu$ with $\left\langle\mu, \phi_{c}\right\rangle<\infty$.

Before reading the remainder of this section, it is recommended that the reader consults Appendix B regarding the definition of time-inhomogeneous superdiffusions as well as the space-time $H$-transform (weighted superdiffusion) introduced there.

Let $X$ be a $(L, \beta, \alpha ; D)$-superdiffusion with $X_{0}=\mu$. Let $H(x, t):=\exp \left(-\lambda_{c} t\right) \phi_{c}(x), x \in D, t \geqslant 0$. It turns out (see Lemma 3 in Appendix B) that the (time-inhomogeneous) process $X^{H}$ defined by

$$
X_{t}^{H}:=H(\cdot, t) X_{t} \quad\left(\text { that is, } \frac{\mathrm{d} X_{t}^{H}}{\mathrm{~d} X_{t}}=H(\cdot, t)\right), \quad t \geqslant 0
$$

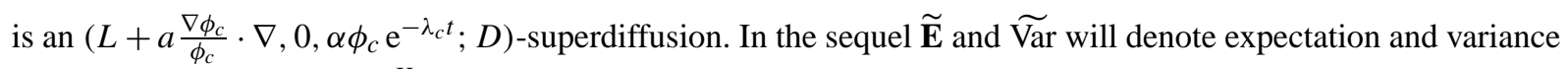
with respect to the law of $X^{H}$.

Lemma 1 (Bounded variance).

$$
\lim _{t \rightarrow \infty} \widetilde{\operatorname{Var}}^{\phi_{c} \mu}\left(\left\|X_{t}^{H}\right\|\right)=\int_{0}^{\infty} \mathrm{d} s \mathrm{e}^{-2 \lambda_{c} s}\left\langle\mu, \mathcal{S}_{s}\left[\alpha \phi_{c}^{2}\right]\right\rangle<\infty,
$$

and $\left\|X^{H}\right\|$ is a uniformly integrable $\widetilde{\mathbf{P}}^{\phi_{c} \mu}$-martingale.

Proof. Let $\bar{X}^{H}$ denote the total mass process, i.e.,

$$
\bar{X}^{H}:=\left\|X^{H}\right\| \text {. }
$$


Abbreviate

$$
L_{0}^{\phi_{c}}:=L+a \frac{\nabla \phi_{c}}{\phi_{c}} \cdot \nabla
$$

and note that in fact

$$
L_{0}^{\phi_{c}}(u)=\phi_{c}^{-1}\left(L+\beta-\lambda_{c}\right)\left(\phi_{c} u\right)=H^{-1}\left(L+\beta+\partial_{t}\right)(H u) .
$$

(Here $\partial_{t}$ denotes differentiation with respect to time.) Let $\mathcal{S}^{\phi_{c}}$ denote the $h$-transformed semigroup with $h=\phi_{c}$, that is $\mathcal{S}_{s}^{\phi_{c}}(\cdot)=\left(\phi_{c}\right)^{-1} \mathcal{S}_{s}\left(\phi_{c} \cdot\right)$.

Define $\mathcal{S}_{s}^{H}:=\mathrm{e}^{-\lambda_{c} s} \mathcal{S}_{s}^{\phi_{c}}$; then the semigroup $\left\{\mathcal{S}_{s}^{H}\right\}_{s} \geqslant 0$ corresponds to the operator $L_{0}^{\phi_{c}}$ that has no zeroth order part. In particular then

$$
\mathcal{S}_{s}^{H} 1 \leqslant 1 \text {. }
$$

Finally, the product-criticality assumption on $L+\beta-\lambda_{c}$ guarantees that the diffusion process corresponding to $L_{0}^{\phi_{c}}$ on $D$ is positive recurrent (ergodic) (see Section 4.4 in Pinsky [15]). (Since ergodicity implies conservativeness, thus in fact $\mathcal{S}_{s}^{H} 1=1$; nonetheless, for us it will be enough to know (13).)

By Lemma 3(b) of Appendix B along with Theorem A2 in [7], we have that for all $f \in C_{\text {const }}^{2}(D):=\{f \in$ $C^{2}(D): \exists \Omega \Subset D$ such that $f=$ const on $D \backslash \Omega$,

$$
\mathrm{d}\left\langle X_{t}^{H}, f\right\rangle=\left\langle X_{t}^{H}, L_{0}^{\phi_{c}} f\right\rangle \mathrm{d} t+\mathrm{d} M_{t}(f),
$$

where $\left\{M_{t}(f)\right\}_{t \geqslant 0}$ is a square-integrable $\widetilde{\mathbf{P}} \phi_{c} \mu$-martingale, and its quadratic variation (i.e. the increasing process in the Doob-Meyer decomposition) $\langle M(f)\rangle$ is given by

$$
\langle M(f)\rangle_{t}=\int_{0}^{t} \mathrm{~d} s \mathrm{e}^{-\lambda_{c} s}\left\langle X_{s}^{H}, \alpha \phi_{c} f^{2}\right\rangle, \quad t \geqslant 0 .
$$

(One can take the function class $C_{\text {const }}^{2}(D)$ instead of just $C_{c}^{2}(D)$, because the diffusion process corresponding to $L_{0}^{\phi_{c}}$ on $D$ is conservative, that is, it never leaves the domain $D$ with probability one.)

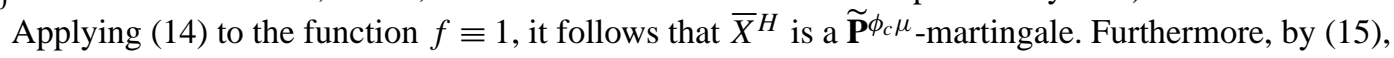

$$
\widetilde{\mathbf{E}}^{\phi_{c} \mu}\left[\left\langle X_{t}^{H}, 1\right\rangle^{2}\right]=\left\langle\mu, \phi_{c}\right\rangle^{2}+\int_{0}^{t} \mathrm{~d} s \mathrm{e}^{-\lambda_{c} s}\left\langle\phi_{c} \mu, \mathcal{S}_{s}^{H}\left[\alpha \phi_{c}\right]\right\rangle .
$$

That is

$$
\widetilde{\operatorname{Var}}^{\phi_{c} \mu}\left(\left\|X_{t}^{H}\right\|\right)=\int_{0}^{t} \mathrm{~d} s \mathrm{e}^{-\lambda_{c} s}\left\langle\phi_{c} \mu, \mathcal{S}_{s}^{H}\left[\alpha \phi_{c}\right]\right\rangle=\int_{0}^{t} \mathrm{~d} s \mathrm{e}^{-2 \lambda_{c} s}\left\langle\mu, \mathcal{S}_{s}\left[\alpha \phi_{c}^{2}\right]\right\rangle .
$$

Letting $t \rightarrow \infty$ we obtain the first statement of the lemma.

Replacing $t$ by $\infty$ in the first of the integrals in (17), we have from (13) and from our assumptions that

$$
\widetilde{\operatorname{Var}}^{\phi_{c} \mu}\left(\left\|X_{t}^{H}\right\|\right) \leqslant \int_{0}^{\infty} \mathrm{d} s \mathrm{e}^{-\lambda_{c} s}\left\langle\phi_{c} \mu, \mathcal{S}_{s}^{H}\left[\alpha \phi_{c}\right]\right\rangle \leqslant \lambda_{c}{ }^{-1}\left\|\alpha \phi_{c}\right\|_{\infty}\left\langle\mu, \phi_{c}\right\rangle<\infty .
$$

Hence, by (16),

$$
\sup _{t \geqslant 0} \widetilde{\mathbf{E}}^{\mu \phi_{c}}\left[\left\langle X_{t}^{H}, 1\right\rangle^{2}\right]<\infty,
$$

and consequently $\bar{X}^{H}$ is uniformly integrable. This completes the proof of the second statement of the lemma. 
Remark 2. Our proof of LLN will indeed use the condition that $\alpha \phi_{c}$ is bounded, however it is quite possible that this condition is not necessary and that assuming the finiteness of the integral in (10) (along with $\left\langle\mu, \phi_{c}\right\rangle<\infty$ ) would suffice.

An immediate consequence of uniform integrability is that $\widetilde{\mathbf{E}}^{\phi_{c} \mu}\left[\bar{X}_{\infty}^{H}\right]=\left\langle\mu, \phi_{c}\right\rangle$, which is finite by assumption, and positive for $\mu \neq 0$. This yields that $\widetilde{\mathbf{P}}^{\phi_{c} \mu}\left[\bar{X}_{\infty}^{H}=0\right]<1$ for $\mu \neq 0$. We record this in a lemma.

Lemma 2 (Limit of the total mass). The martingale $\bar{X}^{H}$ has a $\widetilde{\mathbf{P}}_{c} \phi^{\mu}$-a.s. limit $\bar{X}_{\infty}^{H}:=\lim _{t \rightarrow \infty} \bar{X}_{t}^{H}$ which is positive with positive probability.

\subsection{Heuristics for the Law of Large Numbers}

Before stating the Law of Large Numbers for the class of superdiffusions under consideration, in this subsection we give some heuristic computations. These will justify why we call our main result 'the Law of Large Numbers'.

Working with the $H$-transformed superprocess and at the same time, having the particle approximation in mind, consider particles with underlying motion $Y$ corresponding to the elliptic operator $L_{0}^{\phi_{c}}$ (the probabilities for $Y$ will be denoted by $\left.\left\{\mathbb{P}^{x}, x \in D\right\}\right)$ and with critical binary branching at rate $\exp \left[-\lambda_{c} t\right] \alpha(x)$ at position $x \in D$ and time $t \geqslant 0$. Furthermore let the system be started with initial discrete measure being "close" to $v \neq 0$.

Let $\mathcal{I}_{t}^{n}$ denote the collection of particles alive at time $t$ in the $n$th approximation step. Finally, the event of 'survival' is $\left\{\left|\mathcal{I}_{t}^{n}\right|>0 \forall t>0\right\}$.

The Law of Large Numbers would mean that if $0 \not \equiv f \in C_{c}^{+}(D)$, then as $t \rightarrow \infty$, (and without further specifying what “ $\approx$ " means),

$$
\|v\| \frac{\left(1 /\left|\mathcal{I}_{t}^{n}\right|\right) \sum_{x \in \mathcal{I}_{t}^{n}} f(x)}{\left\langle v, \mathbb{E}^{x}\left[f\left(Y_{t}\right)\right]\right\rangle} \approx 1 \quad \text { on }\left\{\left|\mathcal{I}_{t}^{n}\right|>0 \forall t>0\right\} .
$$

Now recalling that in the $n$th approximating step the individual particle mass is scaled down by $n$ and recalling also Lemma 2, one has that (for $n$ large), $\left|\mathcal{I}_{t}^{n}\right| \approx n \bar{X}_{\infty}^{H}$ as $t \rightarrow \infty$. Putting this together with (18), one gets formally, that for large $n$,

$$
\|v\| \frac{(1 / n) \sum_{x \in \mathcal{I}_{t}^{n}} f(x)}{\left\langle\nu, \mathbb{E}^{x}\left[f\left(Y_{t}\right)\right]\right\rangle} \approx \bar{X}_{\infty}^{H}, \quad \text { as } t \rightarrow \infty .
$$

Note that in fact

$$
\left\langle v, \mathbb{E}^{x}\left[f\left(Y_{t}\right)\right]\right\rangle=\widetilde{\mathbf{E}}^{v}\left\langle X_{t}^{H}, f\right\rangle=\mathrm{e}^{-\lambda_{c} t} \mathbf{E}^{\mu}\left\langle X_{t}, f \phi_{c}\right\rangle
$$

$\left(v=\phi_{c} \mu\right)$. (The first equality can be shown for instance by taking first the particular case $v=\delta_{x}, x \in D$, and using that the two expectations satisfy the same parabolic problem; then integrating with respect to $v(\mathrm{~d} x)$.) Furthermore, passing to the limit (as $n \rightarrow \infty$ ) formally, the numerator of the fraction on the left hand side of (19) becomes

$$
\left\langle X_{t}^{H}, f\right\rangle=\mathrm{e}^{-\lambda_{c} t}\left\langle X_{t}, f \phi_{c}\right\rangle .
$$

Hence, for the new test function $0 \not \equiv \hat{f}:=f \phi_{c} \in C_{c}^{+}(D)$,

$$
\frac{\left\langle X_{t}, \hat{f}\right\rangle}{\mathbf{E}^{\mu}\left\langle X_{t}, \hat{f}\right\rangle} \approx \frac{\bar{X}_{\infty}^{H}}{\|v\|}
$$

\subsection{Main theorem}

Making the intuition of the previous subsection precise, we now state our main result: 
Theorem 1 (Law of Large Numbers). Let $f \in C_{c}^{+}(D)$. If $f \not \equiv 0$ and $\|\mu\| \neq 0$, then

$\lim _{t \rightarrow \infty} \frac{\left\langle X_{t}, f\right\rangle}{\mathbf{E}^{\mu}\left\langle X_{t}, f\right\rangle}=\frac{\bar{X}_{\infty}^{H}}{\left\langle\mu, \phi_{c}\right\rangle}, \quad$ in $\mathbf{P}^{\mu}$-probability

Comparing our theorem with (7), we can now identify the limiting distribution: $N^{\mu}=\bar{X}_{\infty}^{H}$ in law.

Remark 3. One has to be a bit careful though when making heuristic inferences using the particle picture as in the previous subsection.

Obviously, the discrete system in the $n$th approximation step is so that $\liminf _{t \rightarrow \infty}\left|\mathcal{I}_{t}^{n}\right| \geqslant 1$ under survival. That is, $\liminf _{t \rightarrow \infty} \frac{1}{n}\left|\mathcal{I}_{t}^{n}\right|>0$ under survival. Recall that, heuristically (for $n$ large), $\frac{1}{n}\left|\mathcal{I}_{t}^{n}\right| \approx \bar{X}_{\infty}^{H}$ as $t \rightarrow \infty$.

On the other hand, in the recent paper [6] an example of a superprocess is given that satisfies the conditions of our previous theorem and for which

$$
\mathbf{P}^{\mu}\left(\bar{X}_{\infty}^{H}=0 \mid S\right)>0,
$$

where $S$ is the event of survival, $S:=\left\{\left\|X_{t}\right\|>0, \forall t \geqslant 0\right\}$.

Conjecture 1. We conjecture that convergence in probability in (20) can be replaced by almost sure convergence.

We close this section with a remark concerning an old result.

Remark 4. A simple case of a superdiffusion is when $D=\mathbb{R}^{d}, d \geqslant 1, L=\frac{1}{2} \Delta$, with $\alpha, \beta$ positive constants (supercritical super-Brownian motion). Here $\lambda_{c}=\beta$ and

$$
\frac{1}{2} \Delta+\beta-\lambda_{c}=\frac{1}{2} \Delta .
$$

Since $\phi_{c}=\tilde{\phi}_{c} \equiv 1, d \geqslant 1$, the operator $\frac{1}{2} \Delta$ is either critical but not product-critical $(d \leqslant 2)$, or subcritical $(d \geqslant 3)$. Therefore this case is not included in our setup. On the other hand, the corresponding (Strong) Law of large Numbers is well known - at least for the discrete particle systems. Using techniques from Fourier transform theory, Watanabe [18] proved SLLN for branching-Brownian motion in $\mathbb{R}^{d}$ and in certain subdomains of it. It is not clear however if his method can be generalized for more general branching diffusions.

\section{Proof of the result}

The proof is based on two observations. The first one is that the problem can be formulated in terms of $X^{H}$, that is, one can reduce the problem to the investigation of a critical superdiffusion with ergodic motion component and exponentially decaying branching rate (again, recall that $X^{H}$ is an $\left(L+a \frac{\nabla \phi_{c}}{\phi_{c}} \cdot \nabla, 0, \alpha \phi_{c} \mathrm{e}^{-\lambda_{c} t} ; D\right)$-superdiffusion).

The second one is that by considering some large time $t+T$ (where both $t$ and $T$ are large), the changes of $\bar{X}^{H}$ are negligible after time $t$, while the remaining time $T$ is still long enough to distribute the produced mass according to the ergodic flow given by the $H$-transformed migration.

To simplify notation, we will write $W:=X^{H}$ (and, accordingly, $\bar{W}_{\infty}:=\bar{X}_{\infty}^{H}$ ). Denote $v:=W_{0}$. By assumption, $\|v\|=\left\langle\mu, \phi_{c}\right\rangle<\infty$. We need to show that for all $\epsilon>0$,

$$
\lim _{t \rightarrow \infty} \mathbf{P}^{\mu}\left(\left|\frac{\left\langle X_{t}, f\right\rangle}{\mathbf{E}^{\mu}\left\langle X_{t}, f\right\rangle}-\frac{\bar{W}_{\infty}}{\|v\|}\right|>\epsilon\right)=0 .
$$

Recall that $\widetilde{\mathbf{P}}$ denotes the probabilities with respect to the law of $W$. Denoting $\phi_{c}^{-1} f=: g \in C_{c}^{+}(D)$, we rewrite (21) as

$$
\lim _{t \rightarrow \infty} \widetilde{\mathbf{P}}^{v}\left(\left|\frac{\left\langle W_{t}, g\right\rangle}{\widetilde{\mathbf{E}}^{v}\left\langle W_{t}, g\right\rangle}-\frac{\bar{W}_{\infty}}{\|v\|}\right|>\epsilon\right)=0 .
$$


It is easy to check that the limiting invariant density for the diffusion corresponding to $L_{0}^{\phi_{c}}$ is $\phi_{c} \tilde{\phi}_{c}$ (recall the normalization $\left.\int_{D} \mathrm{~d} x \phi_{c}(x) \tilde{\phi}_{c}(x)=1\right)$. Since $Z:=\widetilde{\mathbf{E}}^{v} W$ is just the deterministic $L_{0}^{\phi_{c}}$-flow starting from $v$, therefore

$$
\lim _{t \rightarrow \infty} \widetilde{\mathbf{E}}^{v}\left\langle W_{t}, g\right\rangle=\|v\| \cdot\left\langle\phi_{c} \tilde{\phi}_{c}, g\right\rangle,
$$

and consequently our statement is tantamount to saying that for all $\epsilon>0$,

$$
\lim _{t \rightarrow \infty} \widetilde{\mathbf{P}}^{v}\left(\left|\left\langle W_{t}, g\right\rangle-\left\langle\phi_{c} \tilde{\phi}_{c}, g\right\rangle \bar{W}_{\infty}\right|>\epsilon\right)=0 .
$$

Let $T>0$ and let $Z_{W_{t}}$ denote the deterministic $L_{0}^{\phi_{c}}$-flow starting from the (random) measure $W_{t}$. Then

$$
\widetilde{\mathbf{P}}^{v}\left(\left|\left\langle W_{t+T}, g\right\rangle-\left\langle\phi_{c} \tilde{\phi}_{c}, g\right\rangle \bar{W}_{\infty}\right|>\epsilon\right) \leqslant S_{1}(t)+S_{2}(t, T)+S_{3}(t, T),
$$

where

$$
\begin{aligned}
& S_{1}(t):=\widetilde{\mathbf{P}}^{v}\left(\left|\left\langle\phi_{c} \tilde{\phi}_{c}, g\right\rangle \bar{W}_{\infty}-\left\langle\phi_{c} \tilde{\phi}_{c}, g\right\rangle\left\|W_{t}\right\|\right|>\epsilon / 3\right), \\
& S_{2}(t, T):=\widetilde{\mathbf{P}}^{v}\left(\left|\left\langle\phi_{c} \tilde{\phi}_{c}, g\right\rangle\left\|W_{t}\right\|-\left\langle Z_{W_{t}}(T), g\right\rangle\right|>\epsilon / 3\right) \\
& S_{3}(t, T):=\widetilde{\mathbf{P}}^{v}\left(\left|\left\langle Z_{W_{t}}(T), g\right\rangle-\left\langle W_{t+T}, g\right\rangle\right|>\epsilon / 3\right) .
\end{aligned}
$$

Take $\lim \sup _{t \rightarrow \infty} \lim \sup _{T \rightarrow \infty}$ on both sides of (22). We have

$$
\limsup _{t \rightarrow \infty} \widetilde{\mathbf{P}}^{v}\left(\left|\left\langle W_{t}, g\right\rangle-\left\langle\phi_{c} \tilde{\phi}_{c}, g\right\rangle \bar{W}_{\infty}\right|>\epsilon\right) \leqslant \mathrm{I}+\mathrm{II}+\mathrm{III},
$$

where

$$
\begin{aligned}
& \mathrm{I}:=\limsup _{t \rightarrow \infty} S_{1}(t), \\
& \text { II }:=\limsup _{t \rightarrow \infty} \limsup _{T \rightarrow \infty} S_{2}(t, T), \\
& \text { III := } \limsup _{t \rightarrow \infty} \limsup _{T \rightarrow \infty} S_{3}(t, T) .
\end{aligned}
$$

Now, since $\left\|W_{t}\right\| \rightarrow \bar{W}_{\infty}$ as $t \rightarrow \infty$ a.s.,

$$
\mathrm{I}=\lim _{t \rightarrow \infty} S_{1}(t)=0 \text {. }
$$

Also II $=0$, because for all fixed $t \geqslant 0, \lim _{T \rightarrow \infty} S_{2}(t, T)=0$. (Indeed, for all $\omega \in \Omega, \lim _{T \rightarrow \infty}\left\langle Z_{W_{t}(\omega)}(T), g\right\rangle=$ $\left\langle\phi_{c} \tilde{\phi}_{c}, g\right\rangle\left\|W_{t}(\omega)\right\|$.) Therefore, if we show that

$$
\mathrm{III}=0 \text {, }
$$

then we are done.

In order to do this, use at time $t$ that $W$ is a time-inhomogeneous Markov-process, and then apply Chebysev's inequality:

$$
S_{3}(t, T)=\widetilde{\mathbf{E}}^{\nu} \widehat{\mathbf{P}}^{W_{t}}\left(\left|\left\langle Z_{W_{t}}(T), g\right\rangle-\left\langle W_{t+T}, g\right\rangle\right|>\epsilon / 3\right) \leqslant 9 \epsilon^{-2} \widetilde{\mathbf{E}}^{v} \hat{\sigma}_{W_{t}}^{2}\left\langle W_{T}, g\right\rangle,
$$

where $\widehat{\mathbf{P}}$ is the law of the $\left(L+a \frac{\nabla \phi_{c}}{\phi_{c}} \cdot \nabla, 0, \alpha \phi_{c} \mathrm{e}^{-\lambda_{c}(t+s)} ; D\right)$-superdiffusion (here $t$ is fixed and $s>0$ is time) and $\hat{\sigma}^{2}$ denotes variance.

Let us now recall how the formulae for the first two moments of $\left\langle W_{T}, g\right\rangle$ are obtained: by writing $u_{\theta}(t, x)$ for the solution of the semilinear parabolic evolution equation (corresponding to the superprocess) with initial value $\theta g$, one differentiates (repeatedly) with respect to $\theta$ and sets $\theta=0$. 
For time-homogeneous processes with constant branching rate this is written down in detail in [10], p. 37. Since the derivation of these 'moment formulae' only uses differentiation with respect to $\theta$ (and not $t$ or $x$ ), therefore the proof goes through for the more general setting where coefficients are space-time-dependent.

In our case, from these moment formulae and from (27), one obtains (recall also (13)) that for all $T>0$,

$$
S_{3}(t, T) \leqslant \frac{9}{\epsilon^{2}} \cdot \widetilde{\mathbf{E}}^{v} \int_{0}^{T} \mathrm{~d} s 2 \mathrm{e}^{-\lambda_{c}(t+s)}\left\langle W_{t}, \mathcal{S}_{s}^{H}\left[\alpha \phi_{c}\left(\mathcal{S}_{T-s}^{H} g\right)^{2}\right]\right\rangle \leqslant \frac{C \cdot \widetilde{\mathbf{E}}^{v}\left\|W_{t}\right\|}{\epsilon^{2} \cdot \lambda_{c} \mathrm{e}^{\lambda_{c} t}},
$$

with

$$
C=C\left(\|g\|,\left\|\alpha \phi_{c}\right\|\right):=18\left\|\alpha \phi_{c}\right\| \cdot\|g\|^{2} .
$$

(Note that we have an extra factor 2 relative to [10] - indeed in [10] the non-linear term in the semilinear parabolic evolution equation is $\frac{1}{2} \gamma u^{2}$.) Recall that $\|W\|$ is a $\widetilde{\mathbf{P}}^{v}$-martingale with mean $\|v\|$ and continue (28) with

$$
=\frac{C \cdot\|v\|}{\epsilon^{2} \cdot \lambda_{c} \mathrm{e}^{\lambda_{c} t}}
$$

Since this holds for all $T>0$, thus

$$
\limsup _{T \rightarrow \infty} S_{3}(t, T) \leqslant \frac{C \cdot\|v\|}{\epsilon^{2} \cdot \lambda_{c} \mathrm{e}^{\lambda_{c} t}} .
$$

Letting $t \rightarrow \infty$, one obtains (26), completing our proof.

Remark 5. Personal communication with $\mathrm{T}$. Kurtz revealed the possibility of another, alternative way of completing the argument. Namely, once we know that the total mass of $X^{H}$ converges, we can use a (yet unpublished) spatial version of the so-called lookdown construction given in [3] to show that the 'angular part' of $X^{H}$ converges in distribution. (Note that, conditional on the limit of the total mass, the limit of the angular component is constant.)

\section{Appendix A. The behavior of the process in expectation}

For the cases when $L+\beta-\lambda_{c}$ is subcritical, or critical but $\left\langle\mathrm{d} x, \phi_{c} \tilde{\phi}_{c}\right\rangle=\infty$, Theorem 7(b)(ii) in [16] states that for $g \in C_{c}^{+}$,

$$
\lim _{t \rightarrow \infty} \mathrm{e}^{-\lambda_{c} t} \mathbf{E}^{\mu}\left[\left\langle X_{t}, g\right\rangle\right]=0 .
$$

Note, however, that in the proof there is a glitch: the proof simply refers to Theorem 4.9.9 in [15] which deals with the product-critical case only, and is therefore not applicable for the cases mentioned.

Nevertheless, for the subcritical case, and for $\mu \in \mathcal{M}_{c}(D)$, the statement can be verified by a very simple argument as follows (cf. Theorem 4.9.1 in [15]). First, note that by the first moment formula,

$$
\mathrm{e}^{-\lambda_{c} t} \mathbf{E}^{\mu}\left[\left\langle X_{t}, g\right\rangle\right]=\left\langle\mu,\left[\mathcal{T}_{t} g\right](x)\right\rangle
$$

where $\left\{\mathcal{T}_{t}\right\}_{t \geqslant 0}=\left\{\mathrm{e}^{-\lambda_{c} t} \mathcal{S}_{t}\right\}_{t \geqslant 0}$ denotes the semigroup corresponding to the operator $L+\beta-\lambda_{c}$ on $D$.

Make an $h$-transform now:

$$
\left[\mathcal{T}_{t} g\right](x)=h(x)\left[\mathcal{T}_{t}^{h}(\hat{g})\right](x),
$$

where $\hat{g}:=g h^{-1}$, and pick an $h>0$ satisfying $\left(L+\beta-\lambda_{c}\right) h=0$, to reduce the problem to the proof of

$$
\lim _{t \rightarrow \infty}\left\langle h \mu, \mathbb{E}^{x}\left[\hat{g}\left(\xi_{t}^{h}\right)\right]\right\rangle=0,
$$


where $\xi^{h}$ denotes the diffusion corresponding to $\mathcal{T}^{h}$, that is, to the operator $L_{0}^{h}$ (defined analogously to $L_{0}^{\phi_{c}}$ in $(12)$ with $\phi_{c}$ replaced by $h$ ) and $\mathbb{E}$ denotes the corresponding expectation. (Of course, $h \mu \in \mathcal{M}_{c}(D)$.)

Furthermore, it is enough to show the statement with $\mu:=\delta_{x}, x \in D$, because once we know that, the general statement follows by bounded convergence: $\mathbb{E}^{x}\left[\hat{g}\left(\xi_{t}^{h}\right)\right] \leqslant\|\hat{g}\|$ for all $x \in D$ and $t \geqslant 0$.

In [15], Chapter 4 it is shown that subcriticality is invariant under $h$-transforms, and that the transience of a diffusion is equivalent to the subcriticality of the corresponding elliptic operator. Therefore, in our case, it follows that $\xi^{h}$ is transient. Since $g$ is compactly supported, by an obvious comparison argument, it is enough to show (30) with $g$ replaced by the indicator $1_{B}$, where $B$ is a ball. By transience $1_{B}\left(\xi_{t}^{h}\right) \rightarrow 0$ as $t \rightarrow \infty$ a.s., and the statement follows from this and bounded convergence.

Similarly, the critical but non-product critical case can be reduced to the analogous (but much subtler) problem of showing (30) for a null-recurrent $\xi^{h}$. (Cf. the well known analogous limit theorem for countable state space Markov chains - see e.g. Proposition 5.3 and Corollary 6.39 in [12].) In fact, as mentioned in the notes at the end of Chapter 4 in [15], this result is known in the case when $L$ is symmetric with respect to some reference measure $\rho \mathrm{d} x$ (see [1] or [17]). (Recall that $L$ is symmetric if and only if $b=a \nabla Q$ for some $Q \in C^{2, \eta}(D), \eta \in(0,1]$, and in this case $L$ possesses a self-adjoint extension due to the Friedrichs extension theorem - see Chapter 4 in [15].) Recently Pinchover completed the result by proving it for the general (non-selfadjoint) setting (see [14]).

Consequently, the $\rho>\lambda_{c}$ (over-scaling) part of (6) is immediate. One does not need however the above deep result for the $\rho>\lambda_{c}$ part. Here is a simple alternative proof: using an $h$-transform with an $h>0$ satisfying $\left(L+\beta-\lambda_{c}\right) h=0$, the statement is equivalent to

$$
\lim _{t \rightarrow \infty} \mathrm{e}^{\left(\lambda_{c}-\rho\right) t} \mathbb{E}^{x}\left[g\left(\xi_{t}^{h}\right)\right]=0,
$$

for each $x \in D$, which is true in virtue of the boundedness of $g$.

The $\rho<\lambda_{c}$ (under-scaling) part of (6) is harder, and we are only able to provide the rigorous proof of the somewhat weaker assertion:

$$
\limsup _{t \rightarrow \infty} \exp [-\rho t] \mathbf{E}^{\mu}\left[\left\langle X_{t}, g\right\rangle\right]=\infty .
$$

To this end, denote by $p^{L+\beta}(t ; x, \cdot), x \in D$, the kernel corresponding to the operator $L+\beta$ and note that since $g$ is compactly supported, by an obvious comparison argument, it is enough to prove that

$$
\limsup _{t \rightarrow \infty} \frac{1}{t} \log \int_{B} \mu(\mathrm{d} x) p^{L+\beta}(t ; x, B)=\lambda_{c},
$$

for each $x \in D$, and Borel set $B \Subset D$. Clearly, we may assume that $\|\mu\|=1$. To verify (33), make again an $h$ transform with an $h>0$ satisfying $\left(L+\beta-\lambda_{c}\right) h=0$ on $D$. Then $L+\beta$ transforms into $L_{0}^{h}+\lambda_{c}$. Moreover, since the generalized principal eigenvalue is invariant under $h$-transforms and since $\lambda_{c}\left(L_{0}^{h}+\lambda_{c}\right)=\lambda_{c}\left(L_{0}^{h}\right)+\lambda_{c}$, it follows that $\lambda_{c}\left(L_{0}^{h}\right)=0$. Let $p_{0}^{h}(t, x, \cdot)$ denote the transition measures corresponding to $L_{0}^{h}$. Fix $x \in D$ and $B \Subset D$. Since $h(\cdot) h^{-1}(x) p^{L+\beta}(t, x, \cdot)=\mathrm{e}^{\lambda_{c} t} p_{0}^{h}(t, x, \cdot)$ (see Theorem 4.1.1 in [15]), and since $h$ is bounded between two positive constants on $B$, we have

$$
\limsup _{t \rightarrow \infty} \frac{1}{t} \log \int_{B} \mu(\mathrm{d} x) p_{0}^{h}(t ; x, B)=\limsup _{t \rightarrow \infty} \frac{1}{t} \log \int_{B} \mu(\mathrm{d} x) p^{L+\beta}(t ; x, B)-\lambda_{c} .
$$

Since $\|\mu\|=1$ and $p_{0}^{h}(t ; x, B) \leqslant 1$, the left hand side of (34) is non-positive, giving immediately

$$
\lambda_{+}(x, B):=\limsup _{t \rightarrow \infty} \frac{1}{t} \log \int_{B} \mu(\mathrm{d} x) p^{L+\beta}(t ; x, B) \leqslant \lambda_{c} .
$$

Suppose now that $\lambda_{+}(x, B)<\lambda_{c}$ and pick

$$
c \in\left(-\lambda_{c},-\lambda_{+}(x, B)\right) \text {. }
$$


Then by (34) and (36), along with Fubini's theorem, one obtains

$$
\int_{B} \mu(\mathrm{d} x) \int_{0}^{\infty} \mathrm{e}^{c t} p^{L+\beta}(t, x, B) \mathrm{d} t<\infty .
$$

Hence, for almost every $x \in B$,

$$
G^{(L+\beta+c)}(x, B):=\int_{0}^{\infty} \mathrm{e}^{c t} p^{L+\beta}(t, x, B) \mathrm{d} t<\infty .
$$

It follows from general theory then that $\mathrm{e}^{c t} p^{L+\beta}\left(t, x^{\prime}, B^{\prime}\right)$ is in fact integrable for all $x^{\prime} \in D$ and $B^{\prime} \Subset D$, that is, that the operator $L+\beta+c$ possesses a (minimal positive) Green's function on $D$; however this contradicts to the well known fact that an operator with positive generalized principal eigenvalue does not possess a Green's function. (In our case $\lambda_{c}(L+\beta+c)=\lambda_{c}+c>0$.)

\section{Appendix B. The $H$-transform of superdiffusions}

This section treats a generalization of the $h$-transform for superdiffusions introduced in [7]. The $h$-transform was used in the proofs in [9]. The method used in the present paper however requires the spatial function $0<h=h(x)$ to be replaced by a space-time function $0<H=H(x, t)$. (The reader should not confuse with the space-time harmonic transformation yielding a Girsanov-type change of measure - see e.g. [13].)

We start with the more general definition of a time-inhomogeneous superdiffusion. Let $\tilde{L}$ be an elliptic operator on $D \times \mathbb{R}^{+}$of the form

$$
\tilde{L}:=\frac{1}{2} \nabla \cdot \tilde{a} \nabla+\tilde{b} \cdot \nabla
$$

where the functions $\tilde{a}_{i, j}, \tilde{b}_{i}: D \times \mathbb{R}^{+} \rightarrow \mathbb{R}, i, j=1, \ldots, d$, are $C^{1, \eta}(D)$ (for some $\left.\eta \in(0,1]\right)$ in the space, and continuously differentiable in the time coordinate. Moreover assume that the symmetric matrix $\tilde{a}(x, t):=\left(a_{i, j}(x, t)\right)$ is positive definite for all $x \in D$ and $t \in \mathbb{R}^{+}$.

In addition, let $\tilde{\alpha}, \tilde{\beta}: D \times \mathbb{R}^{+} \rightarrow \mathbb{R}$, be $C^{\eta}(D)$ in the space, and continuously differentiable in the time coordinate. Finally assume that $\tilde{\alpha}$ is positive, and $\tilde{\beta}$ is bounded from above.

Definition 2 (Time-inhomogeneous $(\tilde{L}, \tilde{\beta}, \tilde{\alpha} ; D)$-superdiffusion).

(i) The $(\tilde{L}, \tilde{\beta}, \tilde{\alpha} ; D)$-superdiffusion is a measure-valued (inhomogeneous) Markov process, $\left(X, \mathbf{P}^{\mu, r} ; \mu \in\right.$ $\left.\mathcal{M}_{f}(D), r \geqslant 0\right)$, that is, a family $\left\{\mathbf{P}^{\mu, r}\right\}$ of probability measures where $\mathbf{P}^{\mu, r}$ is a probability on $C([r, \infty))$ and the family is indexed by $\left.\mathcal{M}_{f}(D)\right) \times[0, \infty)$, such that the following holds: for each $g \in C_{b}^{+}(D)$ and $\mu \in \mathcal{M}_{f}(D)$,

$$
\mathbf{E}^{\mu, r}\left[\exp -\left\langle X_{t}, g\right\rangle\right]=\exp -\langle\mu, u(\cdot, r ; t, g)\rangle,
$$

where $u=u(\cdot, \cdot ; t, g)$ is a particular non-negative solution to the backward equation

$$
\begin{aligned}
& -\partial_{r} u=\tilde{L} u+\tilde{\beta} u-\tilde{\alpha} u^{2} \quad \text { in } D \times(0, t), \\
& \lim _{r \uparrow t} u(\cdot, r ; t, g)=g(\cdot) .
\end{aligned}
$$


(ii) To determine the solution $u$ uniquely, use the equivalent forward equation along with the minimality of the solution: fix $t>0$ and introduce the 'time-reversed' operator $\hat{L}$ on $D \times(0, t)$ by

$$
\hat{L}:=\frac{1}{2} \nabla \cdot \hat{a} \nabla+\hat{b} \cdot \nabla
$$

where, for $r \in[0, t]$,

$$
\hat{a}(\cdot, r):=\tilde{a}(\cdot, t-r) \quad \text { and } \quad \hat{b}(\cdot, r):=\tilde{b}(\cdot, t-r) ;
$$

furthermore let

$$
\hat{\beta}(\cdot, r):=\tilde{\beta}(\cdot, t-r) \quad \text { and } \quad \hat{\alpha}(\cdot, r):=\tilde{\alpha}(\cdot, t-r) .
$$

Consider now $v$, the minimal non-negative solution to the forward equation

$$
\begin{aligned}
& \partial_{r} v=\hat{L} v+\hat{\beta} v-\hat{\alpha} v^{2} \quad \text { in } D \times(0, t), \\
& \lim _{r \downarrow 0} v(\cdot, r ; t, g)=g(\cdot) .
\end{aligned}
$$

Then

$$
u(\cdot, r ; t, g)=v(\cdot, t-r ; t, g) .
$$

(See also the remark following this definition.)

Remark 6 (Minimal non-negative solutions for forward equations). In the time-homogeneous case, minimal nonnegative solutions for forward equations have been constructed in Appendix A in [7], and in Section 2 in [8] - the construction uses the approximation of $D$ by compactly embedded subdomains with Dirichlet condition on their boundaries. The construction goes through for the time-inhomogeneous setting.

In [7] and [8] the time interval is $[0, \infty)$ rather than $[0, t]$. However that does not make any difference - in fact the solution on the infinite time interval can be defined by first working on finite time intervals and then showing that they can be taken arbitrarily large without having the solution blown up.

As we will see in Lemma 3(b), one way of defining a time-inhomogeneous superdiffusion is to start with a timehomogeneous one, and then to apply an ' $H$-transform'. In general, the $H$-transform of a time-inhomogeneous superdiffusion is defined as follows. Let $0<H \in C^{2, \eta}(D) \times C^{1, \eta}\left(\mathbb{R}^{+}\right)$and let $X$ be a $(\tilde{L}, \tilde{\beta}, \tilde{\alpha} ; D)$-superdiffusion. We define a new process $X^{H}$ by

$$
X_{t}^{H}:=H(\cdot, t) X_{t} \quad\left(\text { that is, } \frac{\mathrm{d} X_{t}^{H}}{\mathrm{~d} X_{t}}=H(\cdot, t)\right), \quad t \geqslant 0 .
$$

This way one obtains a new superdiffusion, which, in general, is not finite measure-valued but only $\sigma$-finite measure-valued. That is, if $\mathcal{M}(D)$ denotes the family of all (finite or infinite) measures on $D$, then

$$
X_{t}^{H} \in \mathcal{M}_{H}^{(t)}(D):=\left\{v \in \mathcal{M}(D) \mid H(\cdot, t)^{-1} v \in \mathcal{M}_{f}(D)\right\}
$$

(cf. [7], p. 688).

In [7], Section 2, it was shown, that, from an analytical point of view, the (spatial) $h$-transform of the superdiffusion is given by a certain transformation of the corresponding semilinear operator. This remains the case for the space-time $H$-transform.

Lemma 3 ( $H$-transform). Let $X^{H}$ be defined by (44). Then

(a) $X^{H}$ is a $\left(\tilde{L}+\tilde{a} \frac{\nabla H}{H} \cdot \nabla, \tilde{\beta}+\frac{\tilde{L} H}{H}+\frac{\partial_{r} H}{H}, \tilde{\alpha} H ; D\right)$-superdiffusion. 
(b) In particular, if $X$ is a time-homogeneous $(L, \beta, \alpha ; D)$-superdiffusion, and $H$ is of the form

$$
H(x, t):=\mathrm{e}^{-\lambda_{c} t} h(x),
$$

where $\lambda_{c}$ is the principal eigenvalue of $L+\beta$, and $h$ is a positive solution of $(L+\beta) h=\lambda_{c} h$, then $X^{H}$ is a $\left(L+a \frac{\nabla h}{h} \cdot \nabla, 0, \alpha h \mathrm{e}^{-\lambda_{c} t} ; D\right)$-superdiffusion.

Remark 7 (Unbounded $\tilde{\beta}$ 's). As it is already the case with the spatial $h$-transform for superdiffusions, it is possible that the coefficient $\tilde{\beta}$ transforms into a new coefficient that is no longer bounded. In fact this can be the very definition of superdiffusions for certain unbounded $\tilde{\beta}$ 's (see [7], Section 2 for explanation).

Proof of Lemma 3. In order to avoid minor technical inconveniences, we implement the method in [7] (see the second paragraph on p. 689). Namely, we use that the Laplace transition functional restricted to the family of measures $\mathcal{M}_{c}(D)$ and the family of functions $C_{c}^{+}(D)$ uniquely determines a measure-valued Markov process, and we choose working with these smaller spaces rather than replacing $\mathcal{M}_{f}(D)$ and $C_{b}^{+}(D)$ by $H$-dependent spaces.

Pick $v \in \mathcal{M}_{c}(D)$, and $f \in C_{c}(D)$. Define $\mu^{(s)}:=v / H(\cdot, s) \in \mathcal{M}_{c}(D)$, and $g^{(t)}(\cdot):=H(\cdot, t) f(\cdot) \in C_{c}^{+}(D)$. Obviously,

$$
\widetilde{\mathbf{E}}^{v, s}\left[\exp -\left\langle X_{t}^{H}, f\right\rangle\right]=\mathbf{E}^{\mu^{(s)}, s}\left[\exp -\left\langle X_{t}, g^{(t)}\right\rangle\right]
$$

By the log-Laplace equation (40), we can continue with

$$
=\exp -\left\langle\frac{v}{H(\cdot, s)}, u\left(\cdot, s ; t, g^{(t)}\right)\right\rangle .
$$

Consider the operator

$$
\mathcal{A}: C^{2, \eta}(D) \times C^{1, \eta}\left(\mathbb{R}^{+}\right) \mapsto C^{\eta}(D) \times C^{\eta}\left(\mathbb{R}^{+}\right)
$$

defined by

$$
\mathcal{A}(u):=\partial_{s} u+(\tilde{L}+\tilde{\beta}) u-\tilde{\alpha} u^{2} .
$$

Define the $H$-transformed operator $\mathcal{A}^{H}$ in the usual way:

$$
\mathcal{A}^{H}(u):=\frac{1}{H} \mathcal{A}(H u) .
$$

Then a direct computation gives

$$
\mathcal{A}^{H}(u):=\frac{\partial_{s} H}{H} u+\partial_{s} u+\tilde{L} u+\tilde{a} \frac{\nabla H}{H} \cdot \nabla u+\tilde{\beta} u+\frac{\tilde{L} H}{H} u-\tilde{\alpha} H u^{2} .
$$

Another, trivial computation yields that if

$$
v(\cdot, \cdot ; t, f):=u(\cdot, \cdot ; t, H(\cdot, t) f) / H(\cdot, t),
$$

then $v(\cdot, \cdot ; t, f)$ is the solution in (41) with $\mathcal{A}$ replaced by $\mathcal{A}^{H}$, and with the property in Definition 2(ii). Thus the quadruple $(\tilde{L}, \tilde{\beta}, \tilde{\alpha} ; D)$ transforms into the quadruple given in part (a).

Part (b) is straightforward computation.

Remark 8. It is precisely equation (48) that justifies the name ' $H$-transform'; the transformation on the semilinear operator works the same way as Doob's $h$-transform would work on a linear operator. 


\section{Acknowledgement}

The authors are grateful to the anonymous referee for several comments and suggestions that helped to improve the presentation and correctness of the paper.

\section{References}

[1] I. Chavel, L. Karp, Large time behavior of the heat kernel: the parabolic $\lambda$-potential alternative, Comment. Math. Helv. 66 (1991) 541-556.

[2] D.A. Dawson, Measure-valued Markov processes, in: P.L. Hennequin (Ed.), École d'été de probabilités de Saint Flour XXI, 1991, in: Lecture Notes in Math., vol. 1541, Springer-Verlag, Berlin, 1993, pp. 1-260.

[3] P. Donnelly, T.G. Kurtz, Particle representations for measure-valued population models, Ann. Probab. 27 (1) (1999) 166-205.

[4] E.B. Dynkin, Branching particle systems and superprocesses, Ann. Probab. 19 (3) (1991) 1157-1194.

[5] E.B. Dynkin, Diffusions, Superdiffusions and Partial Differential Equations, Amer. Math. Soc. Colloq. Publ., vol. 50, Amer. Math. Soc., Providence, RI, 2002.

[6] J. Engländer, An example and a conjecture concerning scaling limits of superdiffusions, Statist. Probab. Lett. 66 (3) (2004) $363-368$.

[7] J. Engländer, R.G. Pinsky, On the construction and support properties of measure-valued diffusions on $D \subset \mathbb{R}^{d}$ with spatially dependent branching, Ann. Probab. 27 (2) (1999) 684-730.

[8] J. Engländer, R.G. Pinsky, Uniqueness/nonuniqueness for positive solutions to semilinear equations of the form $u_{t}=L u+V u-\gamma u^{P}$ in $R^{n}$, J. Differential Equations 192 (2) (2003) 396-428.

[9] J. Engländer, D. Turaev, A scaling limit theorem for a class of superdiffusions, Ann. Probab. 30 (2) (2002) 683-722.

[10] A. Etheridge, An Introduction to Superprocesses, University Lecture Series, vol. 20, Amer. Math. Soc., Providence, RI, 2000.

[11] K. Fleischmann, J. Swart, Extinction versus exponential growth in a supercritical super-Wright-Fisher diffusion, Stochastic Process. Appl. 106 (1) (2003) 141-165.

[12] J.G. Kemeny, J.L. Snell, A.W. Knapp, Denumerable Markov Chains, Springer-Verlag, 1976.

[13] L. Overbeck, Some aspects of the Martin boundary of measure-valued diffusions, in: Measure-Valued Processes, Stochastic Partial Differential Equations, and Interacting Systems, Amer. Math. Soc., Providence, RI, 1994, pp. 179-186.

[14] Y. Pinchover, Large time behavior of the heat kernel, J. Functional Anal. 206 (1) (2004) 191-209.

[15] R.G. Pinsky, Positive Harmonic Functions and Diffusion, Cambridge University Press, 1995.

[16] R.G. Pinsky, Transience, recurrence and local extinction properties of the support for supercritical finite measure-valued diffusions, Ann. Probab. 24 (1) (1996) 237-267.

[17] B. Simon, Large time behavior of the heat kernel: on a theorem of Chavel and Karp, Proc. Amer. Math. Soc. 118 (2) (1993) $513-514$.

[18] S. Watanabe, Limit theorems for a class of branching processes, in: J. Chover (Ed.), Markov Processes and Potential Theory, Wiley, New York, 1967, pp. 205-232. 